\title{
Simulation Study on the Motion of Magnetic Particles in Silicone Rubber-Based Magnetorheological Elastomers
}

\author{
Zhiqiang Xu $\mathbb{D}^{1,2}$ Heng Wu, ${ }^{1}$ Qiuliang Wang, ${ }^{1}$ Liyin Yi, ${ }^{1}$ and Jun Wang ${ }^{1}$ \\ ${ }^{1}$ School of Mechanical Engineering, Xiangtan University, Xiangtan, China \\ ${ }^{2}$ Engineering Research Center of Complex Tracks Processing Technology and Equipment of Ministry of Education, \\ Xiangtan University, Xiangtan, China
}

Correspondence should be addressed to Zhiqiang Xu; 201721541849@smail.xtu.edu.cn

Received 5 June 2019; Accepted 27 June 2019; Published 18 July 2019

Guest Editor: Xizhong An

Copyright (C) 2019 Zhiqiang Xu et al. This is an open access article distributed under the Creative Commons Attribution License, which permits unrestricted use, distribution, and reproduction in any medium, provided the original work is properly cited.

\begin{abstract}
Magnetorheological elastomer (MRE) is an intelligent composite material and has been widely used in various fields such as vibration reduction and sensing. MRE has an excellent magnetorheological effect through the chaining of its internal magnetic particles. Current studies on MREs mainly focus on the preparation of materials and characterization of mechanical properties. However, very few studies have been conducted on the mechanism of magnetic particle motion during MRE curing. Based on the silicone rubber-based MRE, the motion mechanism of magnetic particles during curing was explored through numerical simulation. First, we analyzed the magnetic force and viscous force of magnetic particles in MRE and discussed the equations of motion of magnetic particles under applied magnetic field. Further, we established a uniform magnetic field model through the finite element method and simulated the motion of two magnetic particles under the magnetic field. Finally, we discussed the effects of particle distribution angles, particle radii, applied magnetic field strength, and distance between particles on particle velocity and displacement. The results show that the distance between particles has the greatest influence on the motion of magnetic particles, and the size of the distance between particles will affect the contact time of the particles, thus affecting the chain formation of magnetic particles in the MRE.
\end{abstract}

\section{Introduction}

Magnetorheological elastomer (MRE) is a smart composite material that consists of a polymeric matrix with embedded micron-sized magnetic particles, then solidifying to obtain an elastic material with variable stiffness under an applied magnetic field $[1,2]$. MRE overcomes the shortcomings of magnetorheological fluid (MRF), such as leakage, easy sedimentation, and instability [3-5]. MRE has a wide range of engineering applications because its variable stiffness characteristics, such as tuning dampers, dampers, sensors, and magnetorheological elastic polishing bodies [6-9]. The excellent mechanical properties of MRE are determined by the internal magnetic particles, which will form a magnetic particle chain parallel to the direction of the magnetic field under applied magnetic field [10]. The anisotropic MRE with different mechanical properties was finally obtained by controlling the strength of the applied magnetic field, and the better the arrangement of particles, the better the mechanical properties of MRE [11]. Therefore, in order to improve the mechanical properties of MRE and make it more widely used in engineering applications, further study on its motion of magnetic particles is essential.

At present, the research on magnetic particles in MRE mainly focuses on the types of magnetic particles, particle size, concentration of particles, and the influence of the applied magnetic field on the magnetic particle chain [1214]. However, there are few studies on the chain formation of magnetic particles in MRE under magnetic field, the existing research generally analyzes the formation of chain by magnetic dipole theory $[15,16]$ or study on the final arrangement of magnetic particle chains by changing the influence of different external conditions [17], and there is less quantitative analysis of magnetic particle motion during MRE curing. In addition, the magnetic particle motion under the applied magnetic field was difficult to observe because of 


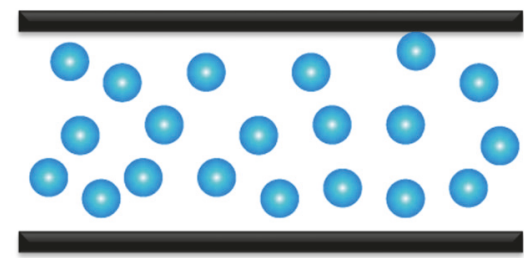

(a)

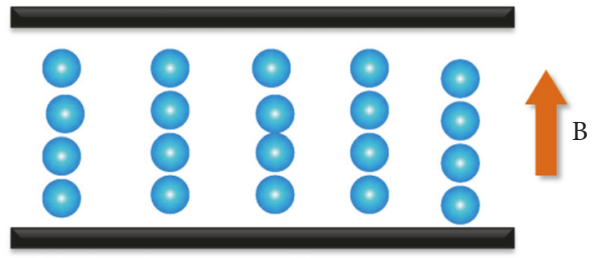

(b)

FIGURE 1: Schematic diagram of chain formation under magnetic field in the curing stage (a) before curing and (b) after curing under magnetic field.

the limitation of particle size, so the motion of the particle was often described by numerical simulation [18-21].

Based on the existing research, the equation of motion of magnetic particles in silicone rubber has been established. The motion of particles under magnetic field was simulated by the finite element method. The formation of magnetic particle chains was explained by the change of velocity and displacement during particle motion, and the effects of two magnetic particles at different distribution angles, particle radius, applied magnetic field, and different distance between the particles on motion process were discussed. Meanwhile, the contact time of magnetic particles under different conditions was analyzed, and the results show that changing the distance between two magnetic particles under the same ratio significantly increases the efficiency of particle chain formation. Moreover, the larger the distance between the particles, the longer the contact time, which provides an effective theoretical basis for preparing a silicone rubberbased MRE with excellent performance.

\section{Theoretical Model}

2.1. Movement Equation. In the preparation process of MRE, anisotropic MRE and isotropic MRE are obtained by controlling the presence or absence of a magnetic field in the curing stage [22]. The magnetic particles in the isotropic MRE are uniformly distributed in the matrix, and the magnetic particles inside the anisotropic MRE form a special chain structure under the magnetic field. Figure 1 is a schematic diagram showing the distribution of magnetic particles of an anisotropic MRE during the curing stage.

Analysis of the force of the magnetic particles in the MRE during the curing stage mainly includes the magnetic force by the applied magnetic field, the viscous force by the silicone rubber matrix, buoyancy, gravity, the interaction force between the particles, and the Brownian force [23]. The magnetic particles produce motion under the combined action of these forces, so the following equation is obtained according to Newton's formula:

$$
m_{i} \frac{d \vec{v}_{i}}{d t}=\vec{F}_{m i}+\vec{F}_{v i}+\vec{F}_{r i}+\vec{F}_{g i}+\vec{F}_{b i}+\vec{F}_{B i}
$$

where $m_{i}$ is the mass of magnetic particle $\mathrm{i}, \vec{v}_{i}$ denotes the particle velocity, $\vec{F}_{m i}$ is the applied magnetic force, $\vec{F}_{v i}$ is the viscous force, $\vec{F}_{r i}$ is the interaction force between particles, and $\vec{F}_{g i}, \vec{F}_{b i}$, and $\vec{F}_{B i}$ represent the gravity, buoyancy, and Brownian forces, respectively.

The magnetic particles used in this study are micronsized particles; thus, gravity and buoyancy can be considered to be balanced; thus, they are not considered. Further, the magnetic and viscous forces of micron-sized particles in incompressible Newtonian liquids are 200 times that of other forces [18]. Therefore, to facilitate calculation for this, the last four terms in (1) are omitted, and only the magnetic and viscous forces of particles moving in the silicone rubber matrix are considered, and the equation of movement of particles can be simplified to

$$
m_{i} \frac{d \vec{v}_{i}}{d t}=\vec{F}_{m i}+\vec{F}_{v i}
$$

In this case, the ordinary differential equation of displacement can be expressed as follows:

$$
\ddot{\vec{u}}=\frac{\vec{F}_{m i}-\vec{F}_{v i}}{m_{i}}
$$

The ordinary differential equation of velocity can also be expressed as follows:

$$
\begin{aligned}
& \dot{\vec{v}}_{i}=\frac{\vec{F}_{m i}-\vec{F}_{v i}}{m_{i}} \\
& \dot{\vec{u}}_{i}=\vec{v}_{i}
\end{aligned}
$$

where $\vec{u}_{i}$ and $\vec{v}_{i}$ represent the velocity and displacement of particle movement, respectively.

2.2. Magnetic Force. The magnetic force on the magnetic particles can be divided into two aspects: one is the force produced by the magnetization of particles under the applied magnetic field and the other is the force between the magnetic dipoles. Assuming that the magnetic particles in the magnetic field are spherical and uniform in size, the magnetic moment of the magnetic particle can be expressed as follows:

$$
\vec{m}=V \vec{M}=\frac{4}{3} \pi R^{3} \chi \vec{H}
$$

where $V$ is the volume of a single particle, $V=\left(4 \mathrm{pi} * R^{\wedge} 3\right) / 3, R$ is the radius, $\vec{M}$ represents magnetization, 


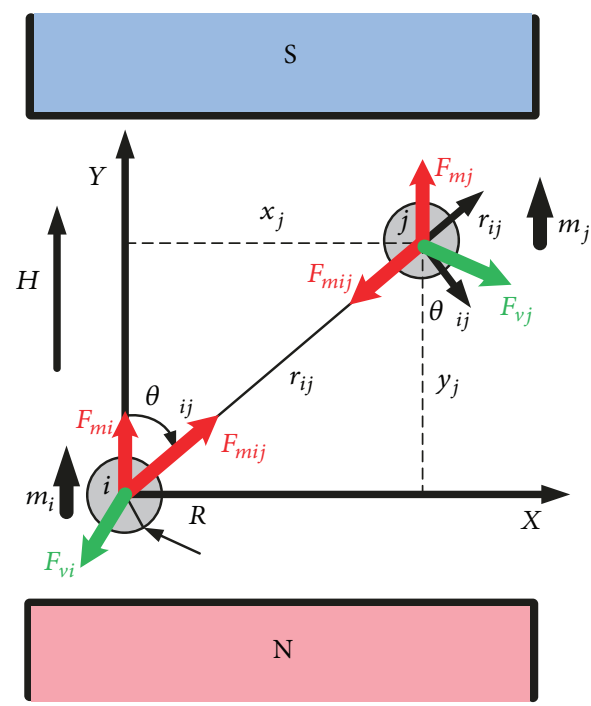

FIGURE 2: Force diagram of particles $\mathrm{i}$ and $\mathrm{j}$ in a 2D plane under applied magnetic field.

$\vec{M}=\chi \vec{H}, \chi$ is the magnetic susceptibility, and $\vec{H}$ is the applied magnetic field strength.

The magnetic force between the magnetic particles $i$ and $\mathrm{j}$ is as follows:

$$
\begin{aligned}
\vec{F}_{m i j} & =\vec{m} \cdot \nabla \vec{B} \\
& =\sum_{j \neq i} \frac{\mu_{0} \vec{m}_{i} \vec{m}_{j}}{4 \pi r_{i j}^{3}}\left[\vec{d}_{i} \cdot \vec{d}_{j}-3\left(\vec{d}_{i} \cdot \widehat{r}_{i j}\right)\left(\vec{d}_{j} \cdot \widehat{r}_{i j}\right)\right]
\end{aligned}
$$

The magnetic force acting on particle $\mathrm{i}$ in the applied magnetic field is as follows:

$$
\vec{F}_{m i}=\mu_{0} \vec{m}_{i} \vec{H}\left(\vec{d}_{i} \cdot \widehat{h}\right)
$$

Based on the above equation, it is concluded that the comprehensive magnetic force of particle $i$ is as follows:

$$
\begin{aligned}
& \vec{F}_{m}^{i}=\vec{F}_{m i j}+\vec{F}_{m i} \\
& \vec{F}_{m}^{i}=\frac{3 \mu_{0} \vec{m}_{i} \vec{m}_{j}}{4 \pi r_{i j}^{4}} \sum_{j \neq i}\left[\left(\vec{d}_{i} \cdot \vec{d}_{j}\right) \widehat{r}_{i j}+\left(\vec{d}_{i} \cdot \widehat{r}_{i j}\right) \vec{d}_{j}\right. \\
& \left.\quad+\left(\vec{d}_{j} \cdot \widehat{r}_{i j}\right) \vec{d}_{i}-5\left(\vec{d}_{i} \cdot \widehat{r}_{i j}\right)\left(\vec{d}_{j} \cdot \widehat{r}_{i j}\right) \widehat{r}_{i j}\right] \\
& \quad+\mu_{0} \vec{m}_{i} \vec{H}\left(\vec{d}_{i} \cdot \hat{h}\right)
\end{aligned}
$$

When only two magnetic particles act, the motion of the particles is a two-dimensional plane motion, so (11) is rewritten:

$$
\vec{F}_{m}^{i}=\frac{4 \pi \mu_{0} \chi^{2} H^{2} R^{6}}{3 r_{i j}^{4}}\left[\left(1-5 \cos ^{2} \theta_{i j}\right) \widehat{r}_{i j}+2 \cos \theta_{i j} \widehat{h}\right]
$$

Then the magnetic field force $\vec{F}_{m}^{i}$ is decomposed in the $\mathrm{x}$ and $\mathrm{y}$ directions and obtained its matrix expression:

$$
\left[\begin{array}{l}
\vec{F}_{m \mathrm{x}}^{i} \\
\vec{F}_{m y}^{i}
\end{array}\right]=\frac{4 \pi \mu_{0} \chi^{2} H^{2} R^{6}}{3 r_{i j}^{4}}\left[\begin{array}{l}
\left(1-5 \cos ^{2} \theta_{i j}\right) \sin \theta_{i j} \\
\left(3-5 \cos ^{2} \theta_{i j}\right) \cos \theta_{i j}
\end{array}\right]
$$

Since the direction of the magnetic force of the particle is related to the angle in the equation (13), so let (1 $\left.5 \cos ^{2} \theta_{i j}\right) \sin \theta_{i j}=0$ and get $\theta_{i j}=\pi / 2$ or $\theta_{i j}=\arccos (\sqrt{15} / 5)$. Therefore, if the particles are to form a particle chain parallel to the direction of the magnetic field, the angle between the two particles $\theta_{i j}<\arccos (\sqrt{5} / 5)$ is required to be satisfied, at this time, and particles appear as close to each. If $\theta_{i j}>$ $\arccos (\sqrt{5} / 5)$, the magnetic particles repel each other.

As shown in Figure 2, $\mu_{0}$ is the vacuum permeability; $\vec{m}_{i}$ and $\vec{m}_{j}$ are the particle magnetic moments; $\vec{d}_{i}$ and $\vec{d}_{j}$ are the direction vectors of the magnetic moment of particles $i$ and $\mathrm{j} ; \widehat{r}_{i j}$ is the direction vectors from particle i to particle $\mathrm{j} ; \vec{H}$ is the direction vector of the applied magnetic field intensity; $\widehat{h}$ is the direction vector of the applied magnetic field intensity; $r_{i j}$ denotes the distance between the centers of two particles $i$ and $j$ and satisfies the following requirement:

$$
r_{i j}=\left|\widehat{r}_{i j}\right|
$$

2.3. Viscous Force. In a static incompressible fluid, the magnetic particles are subjected to the viscous force $\vec{F}_{v}$ of the matrix when they move in the polymer matrix. The particle size is less than $3 \mathrm{~mm}$; thus, according to the Stokes [24],

$$
\vec{F}_{v}=-6 \pi R \eta \frac{d r}{d t}
$$

$R$ is the radius of the particle and $\eta$ is the dynamic viscosity. The selected silicone rubber is purchased from 


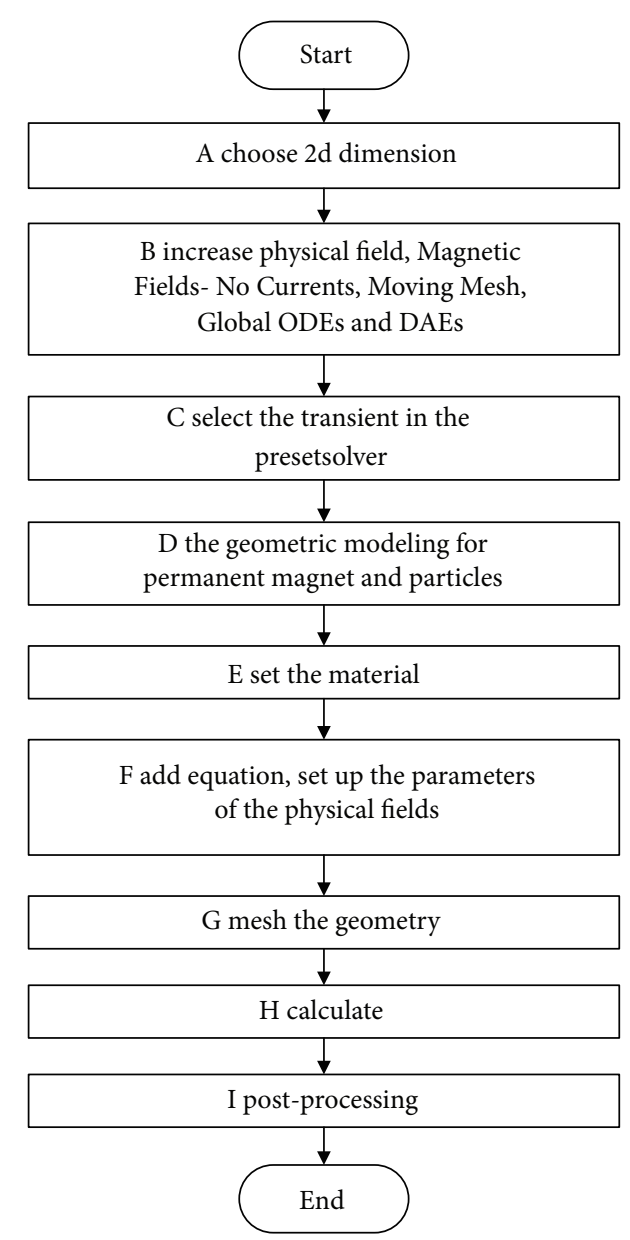

Figure 3: Simulation flow chart.

Dow Corning, USA, and the viscosity is $3.5 \mathrm{~Pa} \cdot \mathrm{s}$ at room temperature. $d r / d t$ is the velocity of the particle motion.

\section{Numerical Simulation}

Based on the force analysis of the magnetic particles in the second section, it is shown that the magnetic particles move under magnetic force and viscous force. In this section, we bring the derived motion equation into the finite element software to simulate the two magnetic particles under the magnetic field, which links the magnetic particle motion theory with the numerical simulation.

First, a two-dimensional plane is selected to establish an empty model, and then the physical field interface is added, including magnetic field no current, moving mesh, and global ordinary differential and differential algebraic equations. Finally, transient research is added to three physics. The magnetic particles under the action of the field are simulated. The magnetic field no current module generates the applied magnetic field required for the movement of the magnetic particles. The moving mesh realizes the movement of the magnetic particles at different time steps. The global ordinary differential and differential algebra equations provide calculations for the velocity and displacement of the particles. The specific flow chart of the simulation is shown in Figure 3.

3.1. Establishment of Permanent Magnetic Field. The applied magnetic field adopts a uniform magnetic field to ensure the accuracy of the calculation result. As shown in Figure 4, the main magnetic field is generated by a pair of horizontally placed permanent magnets, and the magnetic particle moving region is located in the middle of the permanent magnet. Aiming to form a uniform magnetic field having the same magnetic induction intensity in the horizontal and vertical directions, we used a yoke at the end of the magnet, as shown in the red part of Figure 4. The magnetic field design parameters are shown in Table 1.

In order to verify the uniformity of the two-dimensional plane magnetic field, the cut line 1 and the cut line 2 were, respectively, plotted in the horizontal and vertical midpoint positions of the moving region of the particle, and the magnetic flux density on the cut line was calculated. As shown in Figure 5, it can be found that, after the application of the yoke, the variation of the magnetic induction intensity in the moving direction of the particles in the horizontal direction and the vertical direction is remarkably reduced with respect 


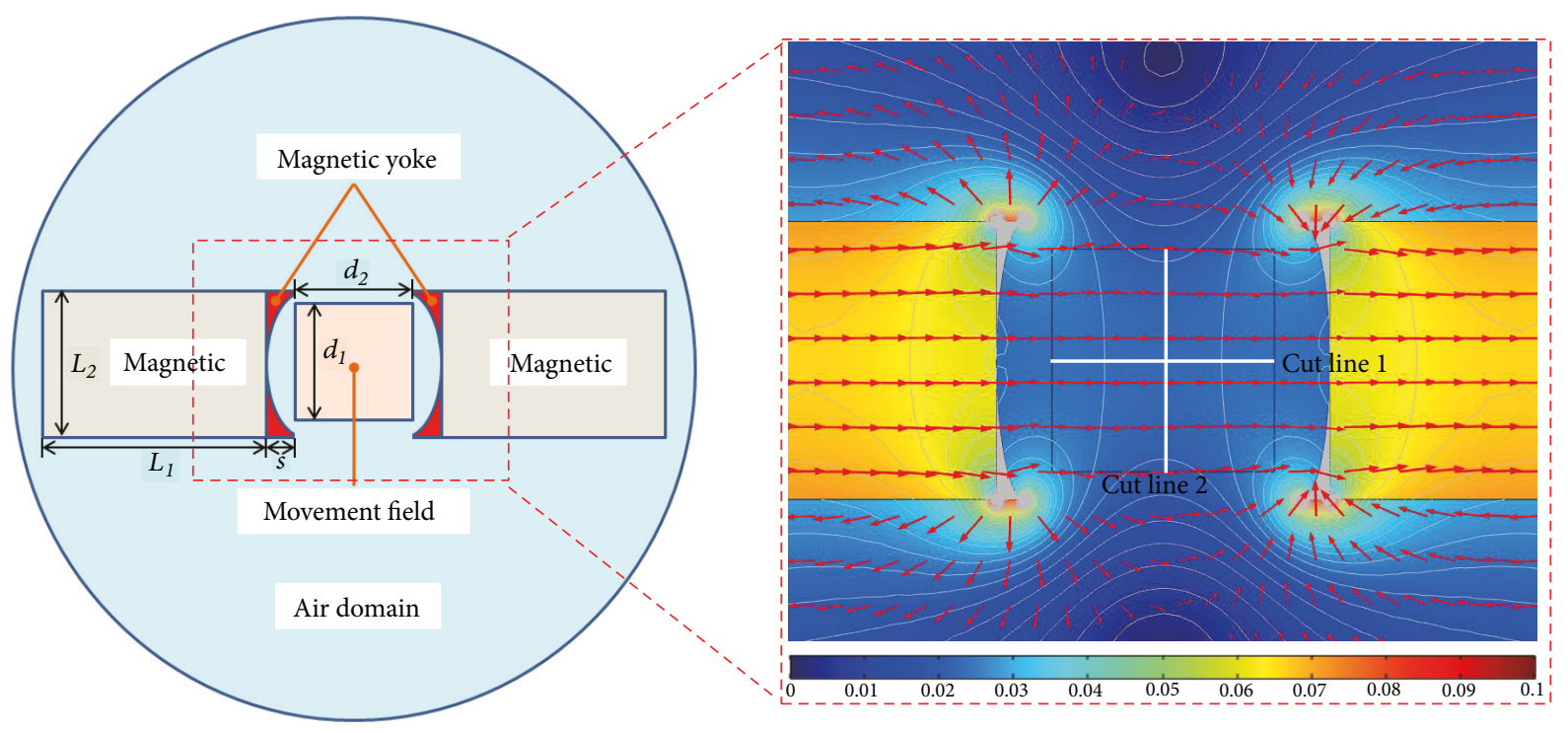

Figure 4: Magnetic field generating device (the uniform magnetic field is generated by a pair of rectangular permanent magnets and yokes, and the middle square area is the moving area of the magnetic particles).

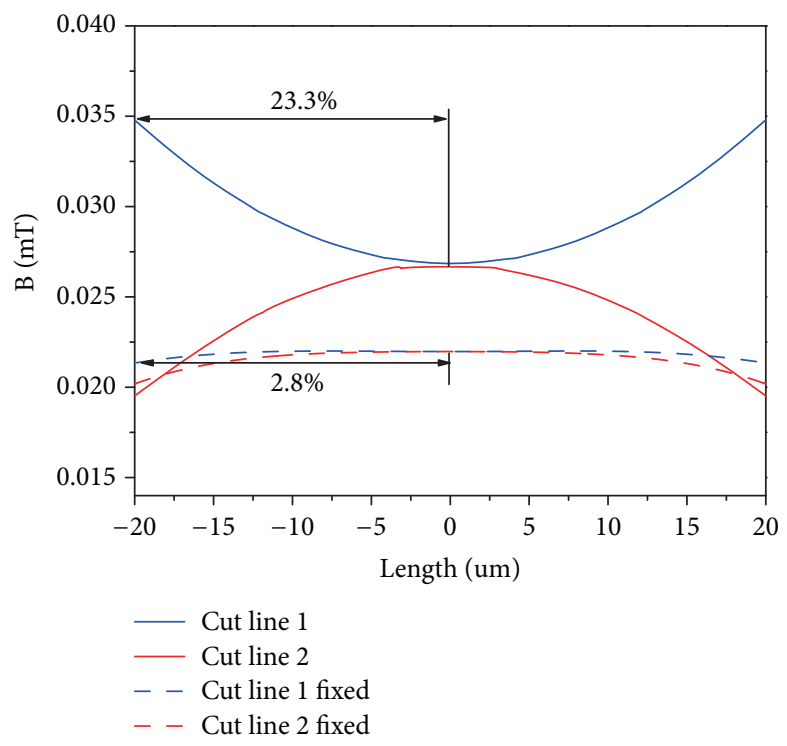

FIGURE 5: The magnitude of the induced intensity on the horizontal and vertical lines (the solid line indicates the distribution of the magnetic field without the yoke, and the dotted line indicates the distribution of the magnetic field with the yoke).

to the nonapplied yoke and thus can be regarded as a uniform magnetic field.

3.2. Simulation Results and Discussion. Since the movement of magnetic particles in MRE directly affects its mechanical properties, the single factor analysis method was used to analyze the influence of four different factors on motion of particles in chain formation, including the distribution angle between two magnetic particles, particle radius, the strength of applied magnetic field, and distance between the particles. In the progress of simulation, the relative magnetic permeability of the particles is 3000 , and the permanent magnetic field described in Section 3.1 is used as the applied magnetic field of the particles.

3.2.1. Influence of Distribution Angles on the Movement of Magnetic Particles. The different types of distribution angles of magnetic particles in the matrix result in different movements of the particles under the magnetic field. In this section, the relationship between the different distribution angles $(\theta)$ of two magnetic particles and their motion parameters was discussed. The angle of distribution was selected to be $0^{\circ}, 30^{\circ}, 60^{\circ}$, and $90^{\circ}$, the applied magnetic field strength is $50 \mathrm{mT}$, the radius of the magnetic particles is $10 \mu \mathrm{m}$, and 


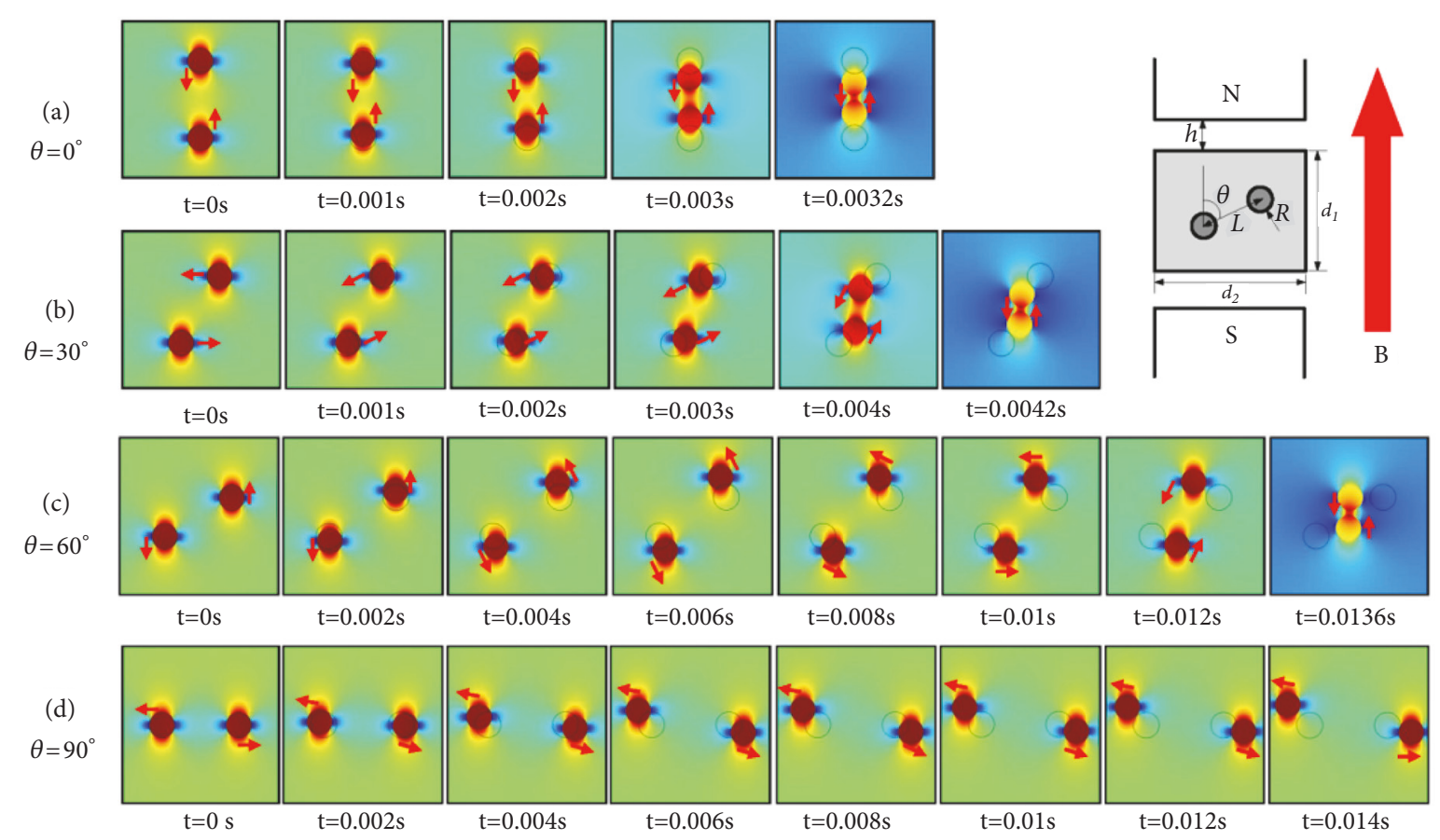

FIGURE 6: Variation of particle movement position with time in different particle distribution angles ((a), (b), (c), and (d) corresponding to the distribution angle between particles $\theta=0^{\circ}, 30^{\circ}, 60^{\circ}$, and $90^{\circ}$ ).

TABLE 1: Simulation parameters of uniform magnetic field.

\begin{tabular}{lcc}
\hline Parameter & Value & Unit \\
\hline Movement field $\left(d_{1} * d_{2}\right)$ & $40 * 40$ & um \\
Magnetic size $\left(L_{1} * L_{2}\right)$ & $80 * 50$ & um \\
Width of magnetic yoke $(\mathrm{s})$ & 10 & um \\
\hline
\end{tabular}

the initial distribution distance of the two particles is $60 \mu \mathrm{m}$. Figure 6 shows the movement of the particles at different times, the black circle in the figure represents the initial distribution of the particles, and the red arrow represents the direction of movement of the particles. From the motion state of the particles, it can be found that the two particles have two effects of attraction and repulsion. When $\theta=0^{\circ}, 30^{\circ}$, and $60^{\circ}$, the particles become mutually attracted, and when $\theta=90^{\circ}$, the particles repel each other; this is because when the angle between the particles is $90^{\circ}$; it is known from (13) that $\theta=90^{\circ}>\arccos (\sqrt{5} / 5)$, so the particles are mutually exclusive.

The three distribution angles of the two particles attracted to each other in Figure 6 were selected, and the changes of velocity and displacement during the movement of the particles were calculated, as shown in (a), (b), and (c) of Figure 7. As shown in Figure 7, the contact time to attract each other is different, where in the corresponding particle contact time is $\theta=0^{\circ}, 30^{\circ}$, and $60^{\circ}$ and $\mathrm{t}=0.0032 \mathrm{~s}, \mathrm{t}=$ $0.0042 \mathrm{~s}$, and $\mathrm{t}=0.0136 \mathrm{~s}$, and as $\theta$ increases, the contact time is longer. As shown in Figures 7(a), 7(b), and 7(c) enlarged view, comparing the velocities of the particles at $t=0.002 \mathrm{~s}$, the average velocity $\mathrm{v}=(\mathrm{v} 1+\mathrm{v} 2) / 2$ corresponding to $\theta=0^{\circ}, 30^{\circ}$, and $60^{\circ}$ gradually decreases, indicating that the smaller $\theta$ is, the larger moving speed of the particles is in the same time, thereby the contact time of the two particles is shorten. At the same time, Figures 7(a), 7(b), and 7(c) show the displacement change of the different distribution angles; as $\theta$ increases, $\mathrm{u} 1 \mathrm{x}$ and $\mathrm{u} 2 \mathrm{x}$ also gradually increases. This is because the particles first move in the $\mathrm{x}$ direction to reduce the angle $\theta$ and then attract each other until the particles contact. Also, it can be seen from the displacements in the y direction that the u1_y and u2_y increase during the movement, indicating that the particles have a vertical movement tendency from the beginning of the movement, and this trend was more obvious when the particles are in contact. Finally, due to the particularity of the grid calculation, when the particle motion stops, the calculation still uses the contact velocity as the final velocity. In fact, the velocity of the particle is 0 at this time, that is, when the velocity of the particle tends to be constant, and it can be regarded as the particles come into contact and the movement stops.

3.2.2. Effect of Different Particle Radius on the Movement of Magnetic Particles. MRE usually use micron-sized magnetic particles, so the radius of particles also is a significant effect 

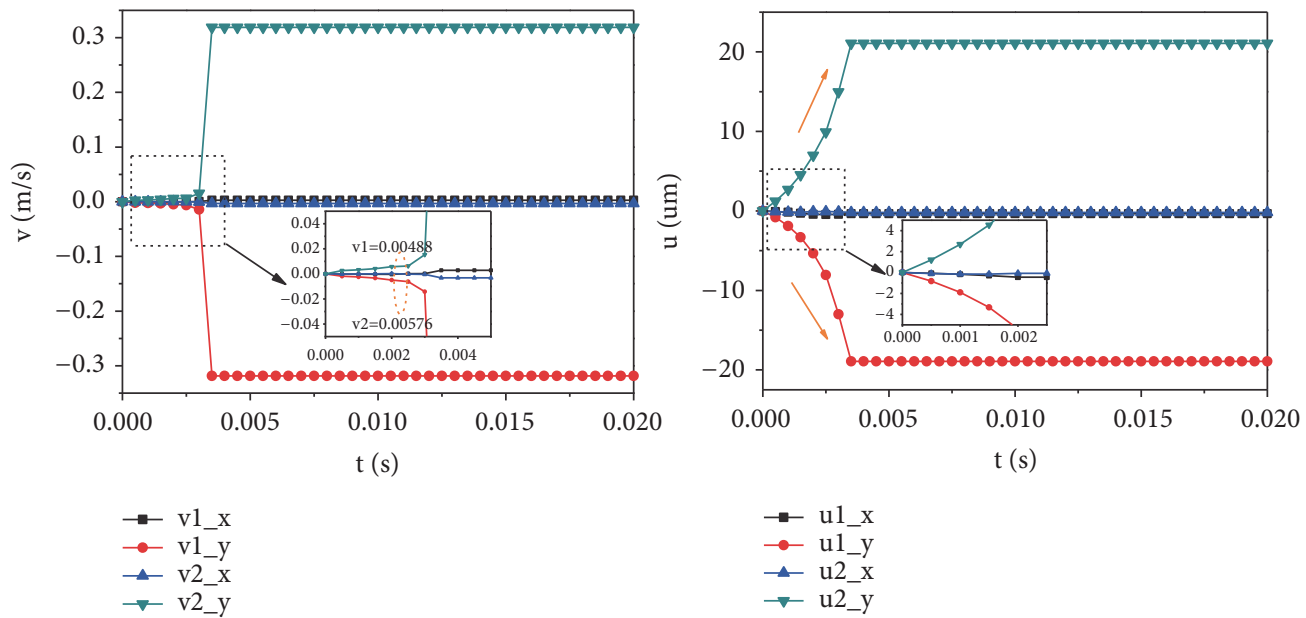

(a)
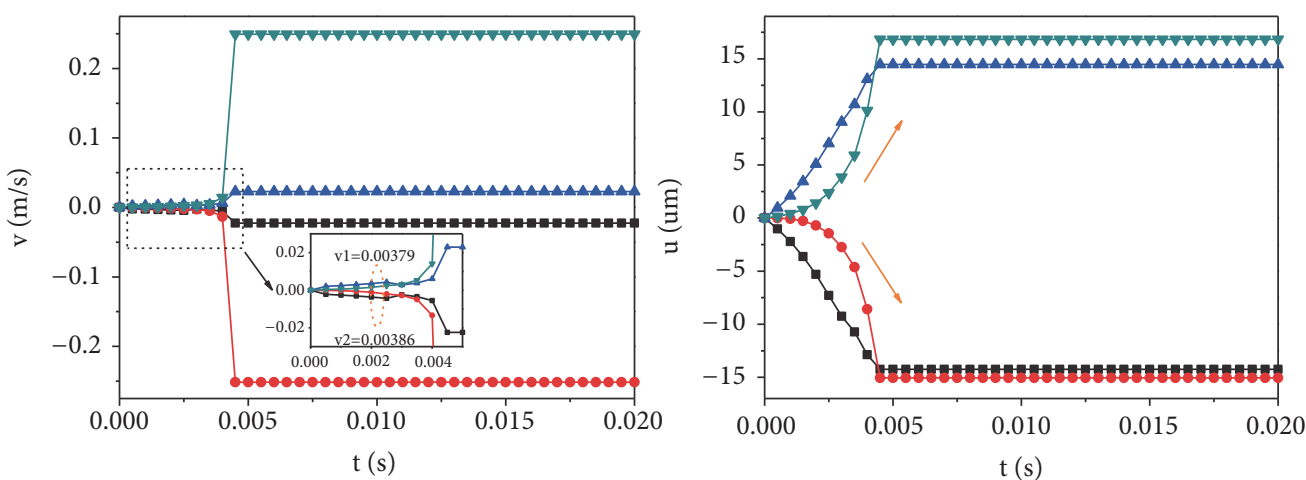

$$
\begin{aligned}
& \rightarrow \mathrm{v} 1 \_x \\
& \rightarrow \mathrm{v} 1-y \\
& -\mathrm{v} 2 \text { - } \\
& \rightarrow \mathrm{v} 2 \text { y }
\end{aligned}
$$

$$
\begin{aligned}
& \text { - u1_x } \\
& \rightarrow \text { u1_y } \\
& \leadsto \text { u2_x } \\
& \rightarrow \text { u2_y }
\end{aligned}
$$

(b)
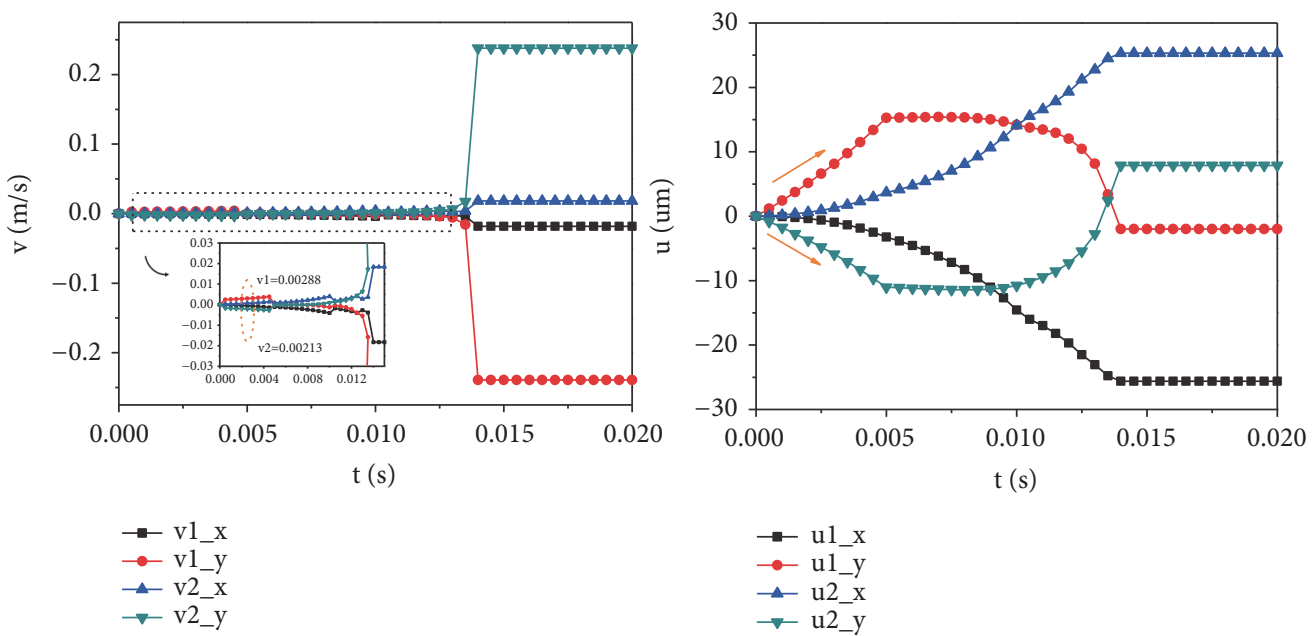

(c)

FIGURE 7: The velocity and displacement of two particles with time at different distribution angles (the two particles corresponding to (a), (b), and (c) are $0^{\circ}, 30^{\circ}$, and $60^{\circ}$, respectively). 

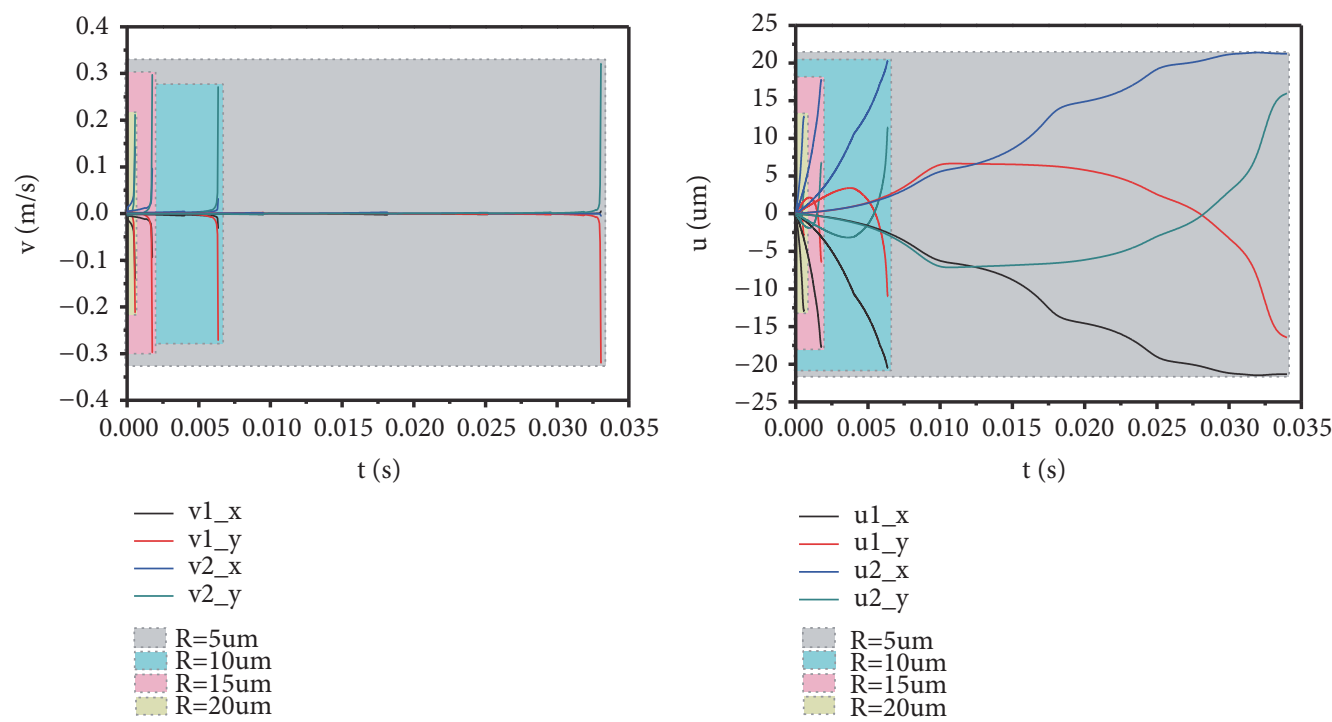

FIGURE 8: Variations of velocity and displacement during the mutual attraction of four different magnetic particle radii.

on the magnetorheological effect of MRE. To analyze the movement of magnetic particles with different radii under magnetic field, 5um, 10um, 15um, and 20um of magnetic particles radius were simulated, and the changes of velocity and displacement parameters during the motion were obtained. In order to consider the influence of the magnetic field in the horizontal direction and the vertical direction, the angle $\theta$ between the particle connection and the applied magnetic field strength was $45^{\circ}$. The distance between the two particles was 60 um.

As shown in Figure 8, the contact time and displacement of the particles become smaller with the larger particles radius; it is because the distance between the particles is constant, with the particle radius increasing, and the corresponding particle volume and mass increase relatively, and then the magnetic force of the particle increases, so that the contact takes less time, which demonstrated that controlling the size of the magnetic particles can effectively control the particle contact time.

\subsubsection{Effect of Different Strength of the Applied Magnetic Field} on Particle Motion. The greater the applied magnetic field strength, the greater the magnetic force of the particles. In order to reflect the influence of different applied magnetic field strength on the movement of magnetic particles, four kinds of uniform magnetic fields were selected in this study. The magnetic field strengths were $50 \mathrm{mT}, 100 \mathrm{mT}, 150 \mathrm{mT}$, and $200 \mathrm{mT}$, respectively, the corresponding magnetic particles have a radius of $5 \mathrm{um}$, the distance between the particles is $30 \mathrm{um}$, and the angle between the particles is $45^{\circ}$.

It can be seen from Figure 9 that the velocity and displacement of the two particles change significantly with the strength of the applied magnetic field increases, the velocity of the particles increases slowly especially at the beginning of the motion, but the particles rapidly increase upon contact; at the same time, the displacement of the two particles in the $\mathrm{x}$ and $\mathrm{y}$ directions gradually increases, indicating that the particles move in parallel and perpendicular to the direction of the magnetic field. When the particles are in contacting, the displacement in the $y$ direction increases rapidly, and the contact of the particles forms a particle chain parallel to the direction of the applied magnetic field. From the contact time of the two particles, the greater the magnetic field strength, the shorter the contact time of the particles. The particle contact time is $0.003 \mathrm{~s}$ in $50 \mathrm{mT}$, while the time is $0.0002 \mathrm{~s}$ in $200 \mathrm{mT}$. That is, the time for the two particles to be chained is reduced by $93 \%$. Because the stronger the magnetic field strength, the stronger the magnetic force of the particles under the same magnetic permeability. It shows that increasing the strength of the applied magnetic field can significantly improve the efficiency of particle contact into the chain.

3.2.4. Effect of Different Distance between Particles on Particle Motion. Due to the random distribution of magnetic particles, there are different distribution distances between the two particles. In this section, four different particle distribution distances were selected, which are $4 * \mathrm{R}, 6 * \mathrm{R}, 8 * \mathrm{R}$, and $10 * \mathrm{R}$. The magnetic field is $50 \mathrm{mT}$, the radius of the magnetic particle $\mathrm{R}$ is $5 \mathrm{um}$, and the angle between the particle connection and the magnetic field is $45^{\circ}$. As shown in Figure 10, the longer the distance between the particles, the longer the contact time of the particles. It can be seen from the displacement curve of the particles that the larger the distance between the particles that the larger the displacement in the $\mathrm{x}$ and $\mathrm{y}$ directions, the longer the contact time.

3.2.5. Effect of Different Conditions on Contact Time of Magnetic Particles. Based on the analysis of the previous sections, the contact time $t$ of the two particles at different angles $(\theta)$, particle radius $(R)$, applied magnetic field strength $(B)$, and the distance $(L)$ between the particles was considered, and the 

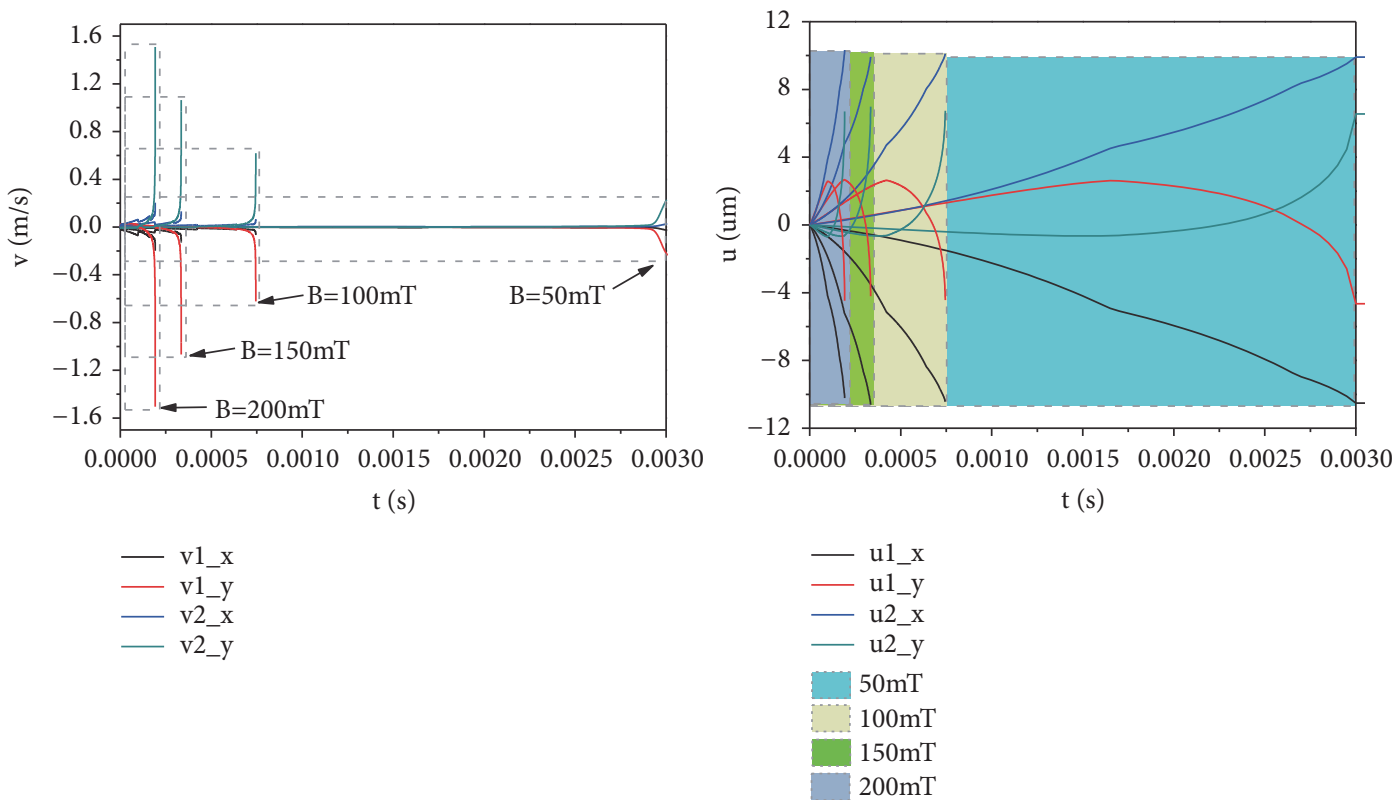

FIGURE 9: Variations in the velocity and displacement of magnetic particles with different applied magnetic field strengths.
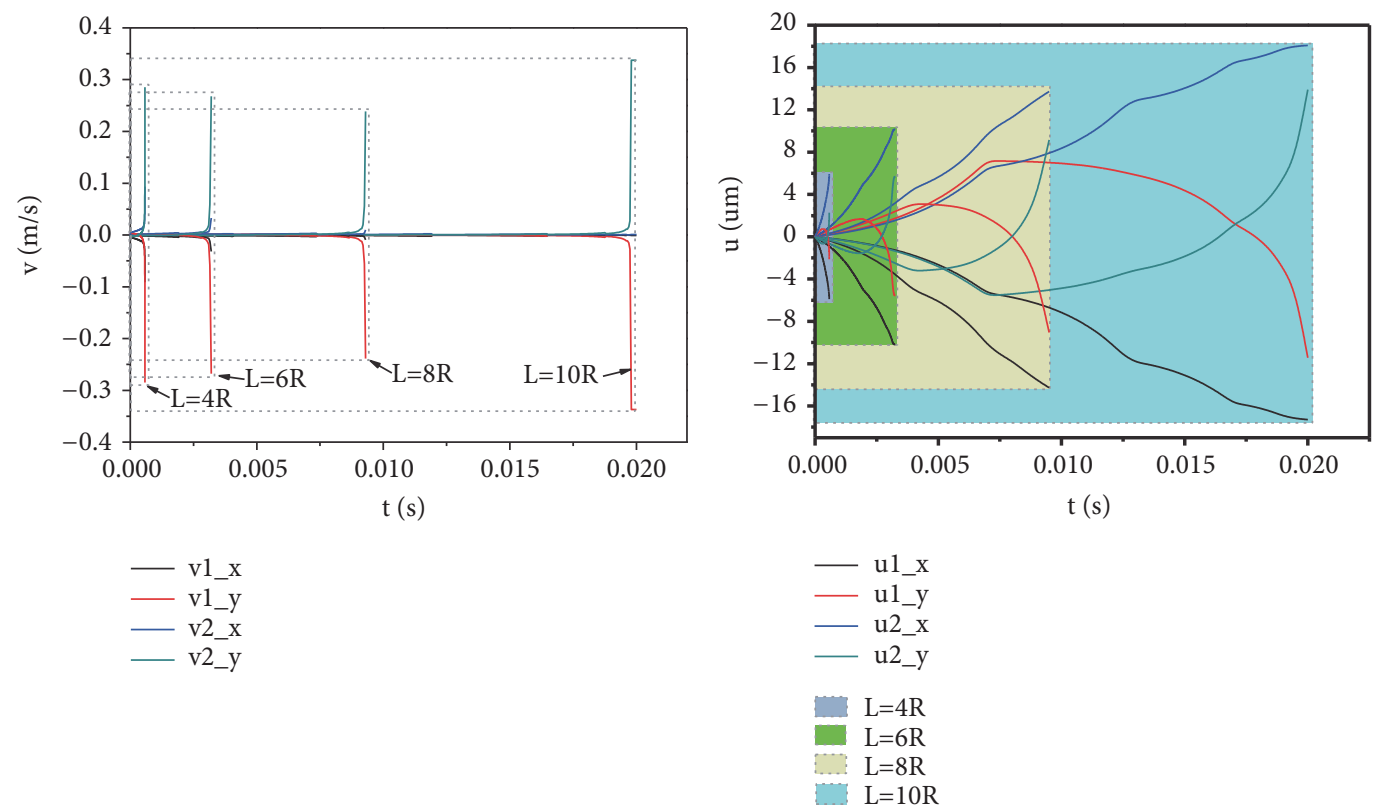

FIGURE 10: Variation of velocity and displacement of particles with time at different particle distribution distances.

influence of the factor on the motion state of the particles was judged. Select initial conditions $\theta_{0}, R_{0}, B_{0}, L_{0}$ for $30^{\circ}, 10 \mathrm{um}$, $100 \mathrm{mT}$, and $50 \mathrm{um}$ and the corresponding scale factor $\lambda=0.5$, $1.0,1.5$, and 2.0 under a single factor. The contact time of each factor under the four scale factors was discussed. The corresponding proportional conditions are shown in Table 2 and the calculation results are shown in Table 3.

According to Table 3, the change of particle contact time under different conditions was obtained, as shown in Figure 11. The height of the histogram reflects the contact time $t$ of the two particles, and the red arrow reflects the change of the contact time of the particles under different ratios. As can be seen from Figure 11, changing the ratio of the four conditions has a significant effect on the contact time of the two particles, comparing the rate of change of particle contact time and the change rate of contact time corresponding to the distance $L$ between the two particles is the largest, reaching $1143 \%$. Secondly, the particle radius $R$, the magnetic field strength $B$ and the distribution angle $\theta$, and the corresponding contact time change rate are $345 \%$, $288 \%$, and $191 \%$, which means that if the particle chain formation efficiency is improved, the particle contact time is 
TABLE 2: Proportional condition setting.

\begin{tabular}{|c|c|c|c|c|}
\hline Factor & $\lambda=0.5$ & $\lambda=1.0$ & $\lambda=1.5$ & $\lambda=2.0$ \\
\hline$\theta\left(^{\circ}\right)$ & $0.5 * \theta_{0}, R_{0}, B_{0}, L_{0}$ & $\theta_{0}, R_{0}, B_{0}, L_{0}$ & $1.5 * \theta_{0}, R_{0}, B_{0}, L_{0}$ & $2 * \theta_{0}, R_{0}, B_{0}, L_{0}$ \\
\hline$R$ (um) & $\theta_{0}, 0.5 * R_{0}, B_{0}, L_{0}$ & $\theta_{0}, R_{0}, B_{0}, L_{0}$ & $\theta_{0}, 1.5 * R_{0}, B_{0}, L_{0}$ & $\theta_{0}, 2 * R_{0}, B_{0}, L_{0}$ \\
\hline$B(\mathrm{mT})$ & $\theta_{0}, R_{0}, 0.5 * B_{0}, L_{0}$ & $\theta_{0}, R_{0}, B_{0}, L_{0}$ & $\theta_{0}, R_{0}, 1.5 B_{0}, L_{0}$ & $\theta_{0}, R_{0}, 2 * B_{0}, L_{0}$ \\
\hline$L(\mathrm{um})$ & $\theta_{0}, R_{0}, B_{0}, 0.5 * L_{0}$ & $\theta_{0}, R_{0}, B_{0}, L_{0}$ & $\theta_{0}, R_{0}, B_{0}, 1.5 * L_{0}$ & $\theta_{0}, R_{0}, B_{0}, 2 * L_{0}$ \\
\hline
\end{tabular}

TABLE 3: Calculated contact time (t).

\begin{tabular}{lllll}
\hline Factor & $\lambda=0.5$ & $\lambda=1.0$ & $\lambda=1.5$ & $\lambda=2.0$ \\
\hline$\theta\left(^{\circ}\right)$ & $4.32 \mathrm{E}-4$ & $5.15 \mathrm{E}-4$ & $7.60 \mathrm{E}-4$ & $1.52 \mathrm{E}-3$ \\
$R(\mathrm{um})$ & $2.90 \mathrm{E}-4$ & $5.15 \mathrm{E}-4$ & $1.03 \mathrm{E}-4$ & $2.65 \mathrm{E}-5$ \\
$B(\mathrm{mT})$ & $2.00 \mathrm{E}-3$ & $5.15 \mathrm{E}-4$ & $2.38 \mathrm{E}-4$ & $1.40 \mathrm{E}-4$ \\
$L(\mathrm{um})$ & $1.59 \mathrm{E}-5$ & $5.15 \mathrm{E}-4$ & $2.38 \mathrm{E}-3$ & $6.42 \mathrm{E}-3$ \\
\hline
\end{tabular}

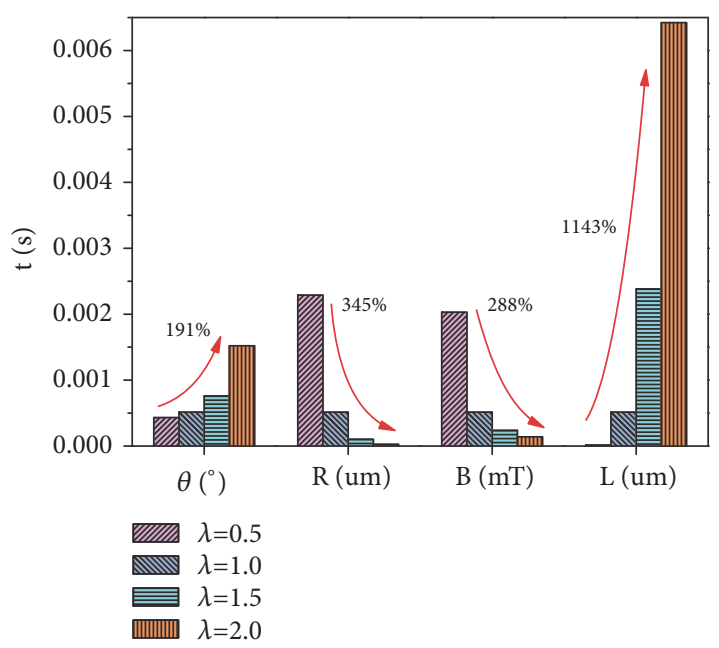

FIGURE 11: Change in contact time of two magnetic particles under different conditions.

shortened; this is achieved by increasing the concentration of the particles to reduce the distance between the particles.

For the magnetic particles in the silicone rubber-based MRE, this section discusses the effects of different distribution angles, particle radii, applied magnetic field strength, and the distance between the particles on the velocity and displacement during mutual attraction, and the results show that these three different conditions have different effects on the movement of the particles. Secondly, the contact time of the particles under different ratios was calculated, and the distance between the particles has the greatest influence on the contact time, and the influence of the particle distribution angle on the contact time of the particles is relatively small.

\section{Conclusions}

In this paper, through the theoretical analysis and simulation calculation of magnetic particles in MRE, the following conclusions are obtained:
(1) The force analysis of the magnetic particles in the silicone rubber-based MRE was carried out, and the motion model of the magnetic particles under the applied magnetic field was established.

(2) The motion of magnetic particles in the MRE were simulated under the uniform magnetic field by the finite element simulation method, and the effects of different particle distribution angles, different particle sizes, different applied magnetic fields, and different particle distances on the velocity and displacement during particle motion were discussed; the simulated result reveal that the distance between particles has the greatest influence on contact time.

(3) The simulation on motion of two magnetic particles provided guiding for optimizing the chaining formation conditions of MRE in the curing process.

\section{Data Availability}

All data included in this study are available upon request by contact with the corresponding author

\section{Conflicts of Interest}

The authors declared no potential conflicts of interest with respect to the research, authorship, and/or publication of this article.

\section{Acknowledgments}

This research was funded by the National Natural Science Foundation of China (grant nos. 51605409 and 51605410) and the Educational Committee Foundation of Hunan (grant no. 17C1531). The authors are grateful for the support.

\section{References}

[1] M. R. Jolly, J. D. Carlson, B. C. Muñoz, and T. A. Bullions, "The magnetoviscoelastic response of elastomer composites 
consisting of ferrous particles embedded in a polymer matrix," Journal of Intelligent Material Systems and Structures, vol. 7, no. 6, pp. 613-622, 1996.

[2] L. Chen, X.-L. Gong, W.-Q. Jiang, J.-J. Yao, H.-X. Deng, and W.$\mathrm{H}$. Li, "Investigation on magnetorheological elastomers based on natural rubber," Journal of Materials Science, vol. 42, no. 14, pp. 5483-5489, 2007.

[3] M. Schümann and S. Odenbach, "In-situ observation of the particle microstructure of magnetorheological elastomers in presence of mechanical strain and magnetic fields," Journal of Magnetism and Magnetic Materials, vol. 441, pp. 88-92, 2017.

[4] N. F. Alias, A. G. Muthalif, K. A. Arpan, and N. D. Nordin, "Experimental investigation of static properties of magnetorheological elastomer," Iranian Journal of Science and Technology Transaction A-Science, pp. 1-13, 2018.

[5] Y. Li, J. Li, W. Li, and H. Du, "A state-of-the-art review on magnetorheological elastomer devices," Smart Materials and Structures, vol. 23, no. 12, Article ID 123001, 2014.

[6] S. B. Kumbhar, S. Chavan, and S. Gawade, "Adaptive tuned vibration absorber based on magnetorheological elastomershape memory alloy composite," Mechanical Systems and Signal Processing, vol. 100, pp. 208-223, 2018.

[7] A. K. Bastola and L. Li, "A new type of vibration isolator based on magnetorheological elastomer," Materials \& Design, vol. 157, pp. 431-436, 2018.

[8] I. Bica, "Magnetoresistor sensor with magnetorheological elastomers," Journal of Industrial and Engineering Chemistry, vol. 17, no. 1, pp. 83-89, 2011.

[9] Z. Xu, Q. Wang, K. Zhu, S. Jiang, H. Wu, and L. Yi, "Preparation and characterization of magnetorheological elastic polishing composites," Journal of Intelligent Material Systems and Structures, vol. 30, no. 10, pp. 1481-1492, 2019.

[10] A. Boczkowska and S. Awietjan, "Microstructure and properties of magnetorheological elastomers," Advanced Elastomers Technology, Properties and Applications, vol. 595, 2012.

[11] K. Danas, S. Kankanala, and N. Triantafyllidis, "Experiments and modeling of iron-particle-filled magnetorheological elastomers," Journal of the Mechanics and Physics of Solids, vol. 60, no. 1, pp. 120-138, 2012.

[12] I. A. Perales-Martínez, L. M. Palacios-Pineda, L. M. LozanoSánchez, O. Martínez-Romero, J. G. Puente-Cordova, and A. Elías-Zúñiga, "Enhancement of a magnetorheological PDMS elastomer with carbonyl iron particles," Polymer Testing, vol. 57, pp. 78-86, 2017.

[13] T. Liu, X. Gong, Y. Xu, S. Xuan, and W. Jiang, "Simulation of magneto-induced rearrangeable microstructures of magnetorheological plastomers," Soft Matter, vol. 9, no. 42, pp. 1006910080, 2013.

[14] J. Li, X. Gong, Z. Xu, and W. Jiang, "The effect of pre-structure process on magnetorheological elastomer performance," International Journal of Materials Research, vol. 99, no. 12, pp. 13581364, 2008.

[15] M. R. Jolly, J. D. Carlson, and B. C. Muñoz, "A model of the behaviour of magnetorheological materials," Smart Materials and Structures, vol. 5, no. 5, pp. 607-614, 1996.

[16] A. Tsutomu, H. Noriyuki, and W. Hitoshi, "Numerical simulation of chainlike cluster movement of feeble magnetic particles by induced magnetic dipole moment under high magnetic fields," Science and Technology of Advanced Materials, vol. 10, Article ID 014609, 2009.
[17] M. Yu, B. X. Ju, J. Fu, X. Liu, and Q. Yang, "Influence of composition of carbonyl iron particles on dynamic mechanical properties of magnetorheological elastomers," Journal of Magnetism and Magnetic Materials, vol. 324, no. 13, pp. 2147-2152, 2012.

[18] Q. Cao, Z. Li, Z. Wang, F. Qi, and X. Han, "Disaggregation and separation dynamics of magnetic particles in a microfluidic flow under an alternating gradient magnetic field," Journal of Physics D: Applied Physics, vol. 51, no. 19, p. 195002, 2018.

[19] A. Sand, J. F. Stener, M. O. Toivakka, J. E. Carlson, and B. I. Pålsson, "A Stokesian dynamics approach for simulation of magnetic particle suspensions," Minerals Engineering, vol. 90, pp. 70-76, 2016.

[20] R. Soda, K. Tanaka, K. Takagi, and K. Ozaki, "Simulationaided development of magnetic-aligned compaction process with pulsed magnetic field," Powder Technology, vol. 329, pp. 364-370, 2018.

[21] M. Hashemi, M. Manzari, and R. Fatehi, "Direct numerical simulation of magnetic particles suspended in a Newtonian fluid exhibiting finite inertia under SAOS," Journal of NonNewtonian Fluid Mechanics, vol. 256, pp. 8-22, 2018.

[22] H. S. Jung, S. H. Kwon, H. J. Choi, J. H. Jung, and Y. G. Kim, "Magnetic carbonyl iron/natural rubber composite elastomer and its magnetorheology," Composite Structures, vol. 136, pp. 106-112, 2016.

[23] U. Banerjee, P. Bit, R. Ganguly, and S. Hardt, "Aggregation dynamics of particles in a microchannel due to an applied magnetic field," Microfluidics and Nanofluidics, vol. 13, no. 4, pp. 565-577, 2012.

[24] S. Krishnamurthy, A. Yadav, P. E. Phelan et al., "Dynamics of rotating paramagnetic particle chains simulated by particle dynamics, Stokesian dynamics and lattice Boltzmann methods," Microfluidics and Nanofluidics, vol. 5, no. 1, pp. 33-41, 2008. 


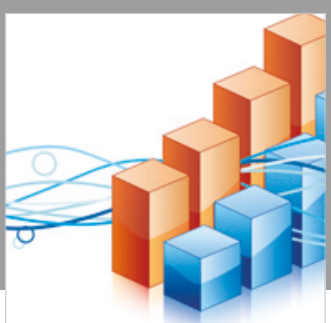

Advances in

Operations Research

\section{-n-m}
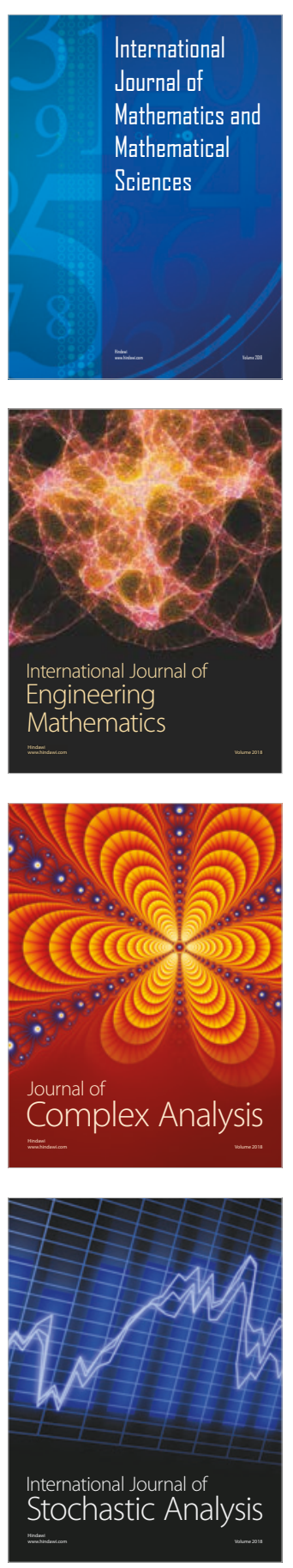
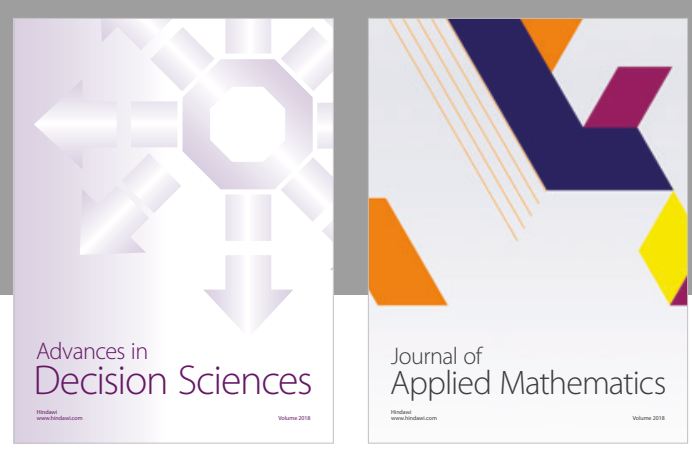

Journal of

Applied Mathematics
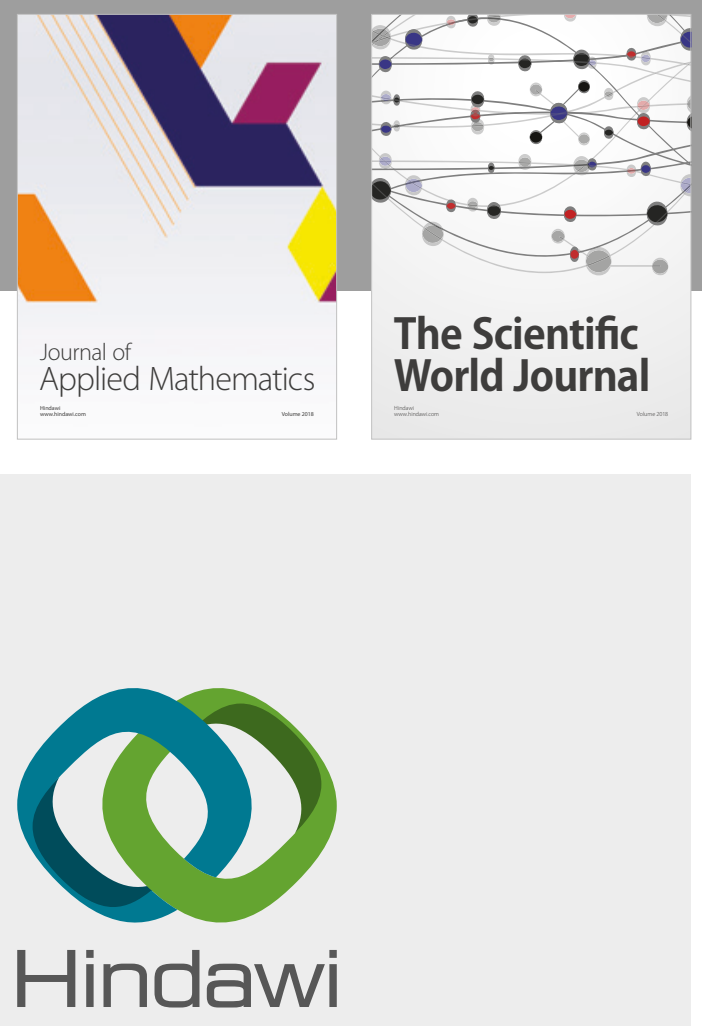

Submit your manuscripts at

www.hindawi.com

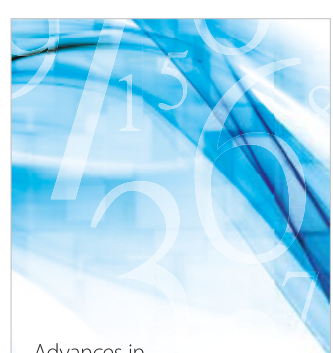

Advances in
Numerical Analysis
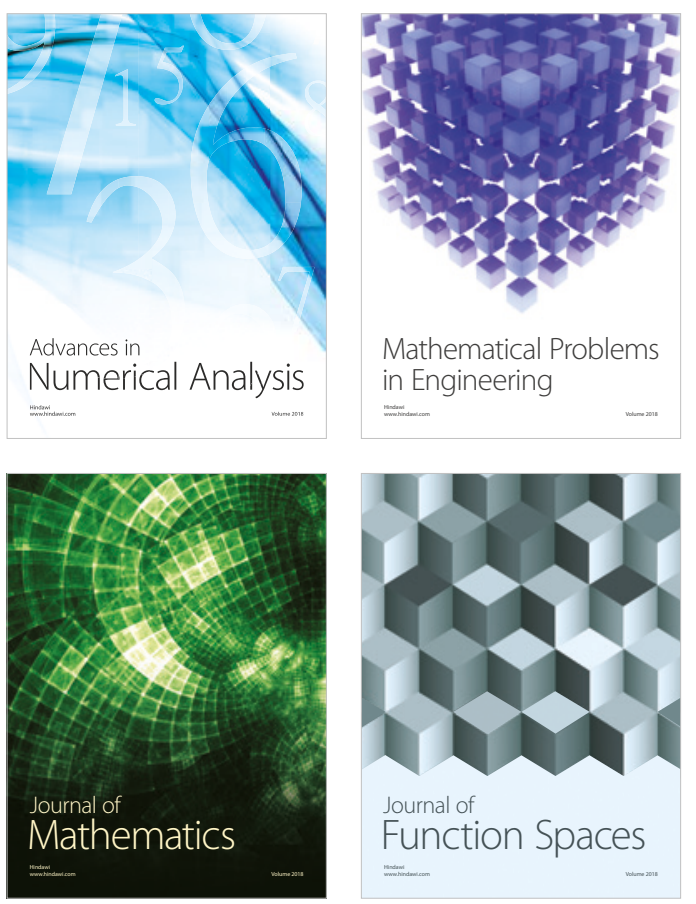

Mathematical Problems in Engineering

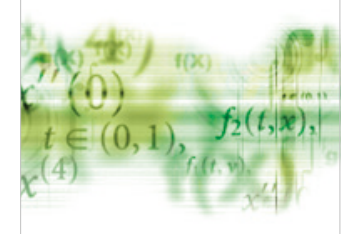

International Journal of

Differential Equations

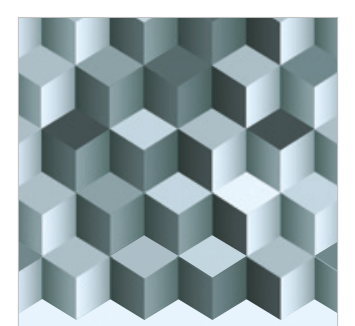

Journal of

Function Spaces

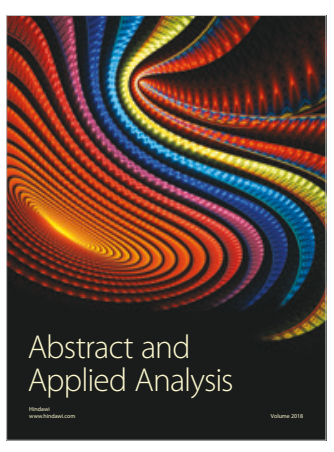

The Scientific

World Journal

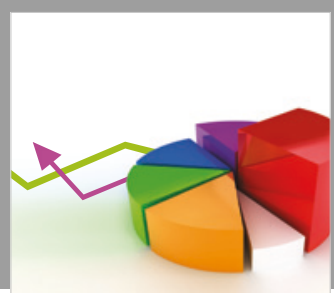

Journal of

Probability and Statistics
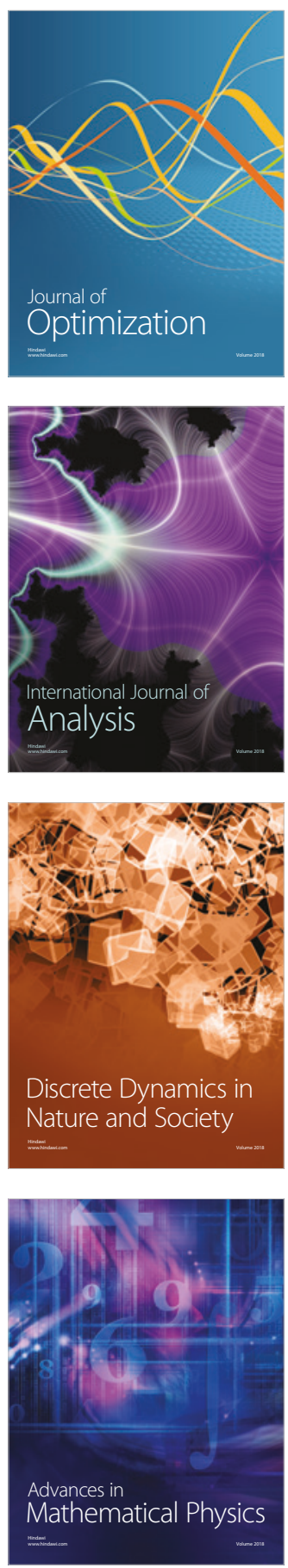